\title{
Non-exponential decay laws in perturbation theory of near threshold eigenvalues
}

\author{
Victor Dinu
}

CAQP, Faculty of Physics, University of Bucharest

P.O. Box MG 11, RO-077125 Bucharest, Romania

Arne Jensen

Department of Mathematical Sciences, Aalborg University

Fr. Bajers Vej 7G, DK-9220 Aalborg Ø, Denmark

Gheorghe Nenciu

CAQP, Faculty of Physics, University of Bucharest

P.O. Box MG 11, RO-077125 Bucharest, Romania

and

Institute of Mathematics of the Romanian Academy

P.O. Box 1-764, RO-014700 Bucharest, Romania

\begin{abstract}
We consider a two channel model of the form

$$
H_{\varepsilon}=\left[\begin{array}{cc}
H_{\mathrm{op}} & 0 \\
0 & E_{0}
\end{array}\right]+\varepsilon\left[\begin{array}{cc}
0 & W_{12} \\
W_{21} & 0
\end{array}\right] \quad \text { on } \quad \mathcal{H}=\mathcal{H}_{\mathrm{op}} \oplus \mathbf{C} .
$$

The operator $H_{\mathrm{op}}$ is assumed to have the properties of a Schrödinger operator in odd dimensions, with a threshold at zero. As the energy parameter $E_{0}$ is tuned past the threshold, we consider the survival probability $\left|\left\langle\Psi_{0}, e^{-i t H_{\varepsilon}} \Psi_{0}\right\rangle\right|^{2}$, where $\Psi_{0}$ is the eigenfunction corresponding to eigenvalue $E_{0}$ for $\varepsilon=0$. We find non-exponential decay laws for $\varepsilon$ small and $E_{0}$ close to zero, provided that the resolvent of $H_{\mathrm{op}}$ is not at least Lipschitz continuous at the threshold zero.
\end{abstract}




\section{Introduction}

In this paper we continue the rigorous study, begun in [13], of the decay laws for resonances produced by perturbation of unstable bound states. There is a large body of literature about decay laws for resonances in general, both at the level of theoretical physics (see e.g. [23], [24], [8], [25], [2], [6], [16], and reference therein) and at the level of rigorous mathematical physics (see e.g. [21], [5], [4], [1], [3], [9], [20], [27], [13], [14], [15], and references therein). The common wisdom, following the computation by Dirac of the decay rate in second order time-dependent perturbation theory (the famous Fermi golden rule), or the Wigner-Weisskopf approximation in scattering theory, is that the decay law is exponential. However it was known for a long time, at least in the semi-bounded case, that the decay law cannot be purely exponential; there must be deviations especially at short and long times (see the physics papers quoted above). So put in more precise terms the question is whether the decay law is quasi-exponential, i.e. is exponential up to error terms, which vanish as the perturbation strength parameter tends to zero. Then the estimation of the error term becomes crucial, and only during the last decades rigorous results were obtained (see the papers quoted above).

The generic result is that (see the papers quoted above for precise formulations) the decay law is indeed quasi-exponential, if the resolvent of the unperturbed Hamiltonian, when projected onto the subspace orthogonal to the considered bound state eigenfunction, is sufficiently smooth in a neighborhood of the unperturbed bound state energy. The problem appears, when the bound state is situated near a threshold, since in this case the resolvent might not be smooth and can even blow up, if there is a resonance at the threshold. At the heuristic level, the fact that this can happen, leads to the possibility of a non-exponential decay law, which has been mentioned (see e.g. [16],[18]) (although this possibility for the single resonance case has been sometimes denied, see [21]).

Having the bound state in the very neighborhood of a threshold is a non-generic situation. But recent advances in experimental technique have made it possible to realize this case experimentally for so-called Feshbach resonances [17], [26]. More precisely, with the aid of a magnetic field it is possible to tune the energy of the bound state continuously through the neighborhood of the threshold energy. As a consequence of applying the magnetic field, the eigenvalue is non-degenerate, and this is the case treated here.

The problem of the decay law for threshold eigenvalues has been considered in [13], [14], [15] under the condition that the shift in the energy due to the perturbation (see (3.1) in [13]) is sufficiently large, such that the 
resonance position is still "far" from the threshold. We proved that in this case the decay law is still (quasi)-exponential, but the Fermi golden rule (i.e. the width of the resonance) has to be modified. In this paper we remove that condition and allow arbitrary location of the resonance by tuning the unperturbed bound state energy across the threshold. Since this can be done experimentally for Feshbach resonances, our conditions on the Hamiltonian are modelled after the Hamiltonians used in Feshbach resonances theory [17], [26].

The main result is that in some energy windows, which depend upon the spectral properties of the unperturbed Hamiltonian at threshold, the decay law is definitely non-exponential for all times. We identify the time scales on which the survival probability varies significantly, as well as the decay law. Also we estimate the errors (vanishing as the perturbation strength parameter tends to zero) giving the first rigorous proof (to the best of our knowledge) of a non-exponential decay law in the perturbative framework.

The contents of the paper is as follows. Section 2 contains the description of the problem and the main results. Section 3 contains the proofs. In Section 4 we display some numerical results on the decay laws obtained. In Appendix A we state some results on resolvent expansions, which imply that Assumption 2.3 is satisfied.

\section{The problem and the results}

We shall develop the theory in a somewhat abstract setting, which is applicable to two channel Schrödinger operators in odd dimensions, as they appear for example in the theory of Feshbach resonances (see e.g. [17], [26], and references therein). The general setting is the one in [13]. Consider

$$
H=\left[\begin{array}{cc}
H_{\mathrm{op}} & 0 \\
0 & H_{\mathrm{cl}}
\end{array}\right]
$$

on $\mathcal{H}=\mathcal{H}_{\mathrm{op}} \oplus \mathcal{H}_{\mathrm{cl}}$. In concrete cases $\mathcal{H}_{\mathrm{op}}=L^{2}\left(\mathbf{R}^{3}\right)$ (or $L^{2}\left(\mathbf{R}_{+}\right)$in the spherically symmetric case) and $H_{\mathrm{op}}=-\Delta+V_{\mathrm{op}}$ with $\lim _{|\mathbf{x}| \rightarrow \infty} V_{\mathrm{op}}(\mathbf{x})=0$. $H_{\text {op }}$ describes the "open" channel. As for the "closed" channel, one starts again with a Schrödinger operator, but with $\lim _{|\mathbf{x}| \rightarrow \infty} V_{\mathrm{cl}}(\mathbf{x})=V_{\mathrm{cl}, \infty}>0$. One assumes that $H_{\mathrm{cl}}$ has bound states below $V_{\mathrm{cl}, \infty}$, which may be embedded in the continuum spectrum of $H_{\mathrm{op}}$. Only these bound states are relevant for the problem at hand. Thus one can retain only one isolated eigenvalue (or a group of almost degenerate eigenvalues isolated from the rest of the spectrum); the inclusion of the rest of the spectrum of $H_{\mathrm{cl}}$ merely "renormalizes" the values of some coefficients, without changing the qualitative picture. In this paper 
we shall consider only non-degenerate eigenvalues, i.e. we shall take $H_{\mathrm{cl}}=E_{0}$ in $\mathcal{H}_{\mathrm{cl}}=\mathrm{C}$, such that

$$
H=\left[\begin{array}{cc}
H_{\mathrm{op}} & 0 \\
0 & E_{0}
\end{array}\right]
$$

on

$$
\mathcal{H}=\mathcal{H}_{\mathrm{op}} \oplus \mathbf{C}=\left\{\Psi=\left[\begin{array}{l}
\psi \\
\beta
\end{array}\right] \mid \psi \in \mathcal{H}_{\mathrm{op}}, \beta \in \mathbf{C}\right\}
$$

Apart from the spectrum of $H_{\mathrm{op}}, H$ has a bound state

$$
\Psi_{0}=\left[\begin{array}{l}
0 \\
1
\end{array}\right], \quad \text { such that } H\left[\begin{array}{l}
0 \\
1
\end{array}\right]=E_{0}\left[\begin{array}{l}
0 \\
1
\end{array}\right] .
$$

The problem is to study the fate of $\Psi_{0}$, when an interchannel perturbation

$$
\varepsilon W=\varepsilon\left[\begin{array}{cc}
0 & W_{12} \\
W_{21} & 0
\end{array}\right]
$$

is added to $H$, i.e. the total Hamiltonian is

$$
H_{\varepsilon}=H+\varepsilon W
$$

Throughout the paper we assume without loss of generality that $\varepsilon>0$. For simplicity we assume that $W$ is a bounded self-adjoint operator on $\mathcal{H}$.

More precisely, the quantity to be studied is the so-called survival probability amplitude

$$
A_{\varepsilon}(t)=\left\langle\Psi_{0}, e^{-i t H_{\varepsilon}} \Psi_{0}\right\rangle .
$$

The first thing to do is to write down a workable formula for $A_{\varepsilon}(t)$. For this purpose we use the Stone formula to express the compressed evolution in terms of the compressed resolvent, and then we use the Schur-LivsicFeshbach-Grushin (SLFG) partition formula to express the compressed resolvent as an inverse (for details, further references, and historical remarks about the SLFG formula, we send the reader to [13]). More precisely, by using the Stone formula and the SLFG formula, one arrives at the following basic formula for $A_{\varepsilon}(t)$, which often appears in the physics literature and is a particular case of the general formula in [13].

$$
A_{\varepsilon}(t)=\lim _{\eta \searrow 0} \frac{1}{\pi} \int_{-\infty}^{\infty} e^{-i t x} \operatorname{Im} F(x+i \eta, \varepsilon)^{-1} d x
$$

with

$$
F(z, \varepsilon)=E_{0}-z-\varepsilon^{2} g(z),
$$


where

$$
g(z)=\left\langle\Psi_{0}, W Q^{*}\left(H_{\mathrm{op}}-z\right)^{-1} Q W \Psi_{0}\right\rangle,
$$

and $Q$ is the orthogonal projection on $\mathcal{H}_{\text {op }}$, considered as a map from $\mathcal{H}$ to $\mathcal{H}_{\text {op }}$.

Since we are interested in the form of $A_{\varepsilon}(t)$, when $E_{0}$ is near a threshold of $H_{\mathrm{op}}$, we shall assume that 0 is a threshold of $H_{\mathrm{op}}$ and that $E_{0}$ is close to zero.

Assumption 2.1. (i) There exists $a>0$, such that $(-a, 0) \subset \rho\left(H_{\mathrm{op}}\right)$ (the resolvent set) and $[0, a] \subset \sigma_{\mathrm{ess}}\left(H_{\mathrm{op}}\right)$.

(ii) $\left|E_{0}\right| \leq \frac{1}{2}$.

From Assumption 2.1 and (2.8) we get the following result.

Proposition 2.2. (i) $g(z)$ is analytic in $\mathbf{C} \backslash\{(-\infty,-a] \cup[0, \infty)\}$.

(ii) $g(\bar{z})=\overline{g(z)}$.

(iii) $g(z)$ is strictly increasing on $(-a, 0)$.

(iv) $\operatorname{Im} g(z)>0$ for $\operatorname{Im} z>0$.

For $E_{0}$ outside a small ( $\varepsilon$-dependent) neighborhood of the origin the situation is well understood, both at the heuristic level, and at the rigorous level. Indeed, for negative $E_{0}$, using the analytic perturbation theory, one can show that

$$
\left|A_{\varepsilon}(t)-e^{-i t E_{\varepsilon}}\right| \lesssim \varepsilon^{2},
$$

where $E_{\varepsilon}$ is the perturbed eigenvalue, which coincides with $E_{0}$ in the limit $\varepsilon \rightarrow 0$. As a consequence the survival probability remains close to one uniformly in time.

Note that in $(2.9)$ we have introduced the notation $\lesssim$, which means less than or equal to, up to a constant, which is irrelevant for our computations. We will use this notation throughout the paper.

On heuristic grounds, if $E_{0}$ is positive, i.e. embedded in the essential spectrum of $H_{\mathrm{op}}, \Psi_{0}$ turns into a metastable decaying state, and the main problem is to compute (up to error terms vanishing in the limit $\varepsilon \rightarrow 0$ ) the "decay law", i.e. $\left|A_{\varepsilon}(t)\right|^{2}$. For eigenvalues embedded in the continuum spectrum the heuristics for the exponential decay law $\left|A_{\varepsilon}(t)\right|^{2} \cong e^{-2 \Gamma(\varepsilon) t}$ runs as follows. Suppose $F(z, \varepsilon)$ is sufficiently smooth, as $z$ approaches the real line from above, $F(x+i 0, \varepsilon)$, for $x$ in a neighborhood of $E_{0}$. Let $F(x+i 0, \varepsilon)=$ $R(x)+i I(x)$. Then the equation $R(x)=0$ has a solution $x_{0}(\varepsilon)$ nearby $E_{0}$. The idea is that the main contribution to the integral in (2.6) comes from the neighborhood of $x_{0}(\varepsilon)$, and in this neighborhood $F(x+i 0, \varepsilon) \cong$ 
$x_{0}(\varepsilon)-x+i I\left(x_{0}(\varepsilon)\right)$, and then

$$
\operatorname{Im} F(x+i \eta, \varepsilon)^{-1} \cong \frac{-I\left(x_{0}(\varepsilon)\right)}{\left(x-x_{0}(\varepsilon)\right)^{2}+I\left(x_{0}(\varepsilon)^{2}\right)},
$$

i.e. it has a Lorenzian peak shape leading to $\left|A_{\varepsilon}(t)\right|^{2} \cong e^{-2 \mid I\left(x_{0}(\varepsilon) \mid t\right.}$. For mathematical substantiation of this heuristics in the case, where $x_{0}(\varepsilon)$ is sufficiently "far" from the origin $\left(x_{0}(\varepsilon) \gtrsim \varepsilon\right)$, we send the reader to [13] and references therein. The problem with the energies near the threshold is that $F(x+i 0, \varepsilon)$ might not be smooth and can even blow up, if the open channel has a resonance at the threshold.

The aim of this paper is to consider the problem of a non-exponential decay law, for the case that $E_{0}$ is near the threshold, at the rigorous mathematical physics level. To this end we need assumptions about the behavior of the function $g(z)$ in the neighborhood of the origin. In stating this assumption we use the notation from [13] to facilitate reference to that paper.

Assumption 2.3. For $\operatorname{Re} \kappa \geq 0$ and $z \in \mathbf{C} \backslash[0, \infty)$ we let

$$
\kappa=-i \sqrt{z}, \quad z=-\kappa^{2} .
$$

Let for $a>0$

$$
D_{a}=\{z \in \mathbf{C} \backslash[0, \infty)|| z \mid<a\}
$$

Then for $z \in D_{a}$

$$
\begin{gathered}
g(z)=\sum_{j=-1}^{2} \kappa^{j} g_{j}+\kappa^{3} r(\kappa), \\
\frac{d}{d z} g(z)=-\frac{1}{2 \kappa} \sum_{j=-1}^{2} \kappa^{j-1} g_{j}+\kappa s(\kappa), \\
\sup _{z \in D_{a}}\{|r(\kappa)|,|s(\kappa)|\}<\infty
\end{gathered}
$$

As already explained, Assumption 2.3 includes the case, when $H_{\mathrm{op}}=$ $-\Delta+V_{o p}$ in odd dimensions. The expansions for the resolvent of $-\Delta+V_{o p}$ leading to (2.12) are provided in [11], [10], [22], [12], [13], [14]. Taking into account that (at least formally)

$$
\frac{d}{d z} g(z)=\left\langle\Psi_{0}, W Q^{*}\left(H_{\mathrm{op}}-z\right)^{-2} Q W \Psi_{0}\right\rangle
$$

(2.13) can be derived in the same manner. More precisely, it can be shown that the expansion (2.12) is differentiable, see [11], [22]. Examples of expansions with the corresponding explicit expressions for coefficients $g_{j}$ are given in the Appendix, with references to the literature. 
We divide the considerations into three cases.

(i) The singular case, in which $g_{-1} \neq 0$. In the Schrödinger case this corresponds to the situation, when $H_{\mathrm{op}}$ has a resonance at the threshold (see the Appendix for details). Let us recall that the free particle in one dimension belongs to this class. From Proposition 2.2(iv) follows that

$$
g_{-1}>0
$$

(ii) The regular case, in which $g_{-1}=0$ and $g_{1} \neq 0$. We note that $g_{-1}=0$ is the generic case for Schrödinger operators in one and three dimensions. Again from Proposition 2.2(iv) one has

$$
g_{1}<0 \text {. }
$$

Let us remark that the behavior $\operatorname{Im} g(x+i 0) \sim x^{1 / 2}$ as $x \rightarrow 0$ is nothing but the famous Wigner threshold law [26], [19].

(iii) The smooth case, in which $g_{-1}=g_{1}=0$. This case occurs for free Schrödinger operators in odd dimensions larger that three, and in the spherical symmetric case for partial waves $\ell \geq 1$. Notice that in this case $\frac{d}{d z} g(z)$ is uniformly bounded in $D_{a}$.

In the first two cases we shall approximate

$$
F(z, \varepsilon)=H(z, \varepsilon)+\text { error term, }
$$

compute the survival probability amplitude from $H(z, \varepsilon)$, and estimate the error term. The main result is that for energies of order $\sim \varepsilon^{4 / 3}$ in the singular case, and $\sim \varepsilon^{4}$ in the regular case, the decay law is not exponential for any significant time interval. Moreover, we shall derive explicit formulae for the survival probability amplitude in terms of the expansion coefficients of $g(z)$. As noted above (see the Appendix), these coefficients can be computed explicitly in a number of interesting cases.

For the smooth case the results in [13] imply that the decay law is always exponential for a significant time interval, or equivalently, for sufficiently small $\varepsilon$, substantiating an heuristic argument in [16].

\subsection{The singular case: $g_{-1}>0$.}

In this case we choose

$$
H_{s}(z, \varepsilon)=E-z-\varepsilon^{2} g_{-1} \kappa^{-1}
$$

where

$$
E=E_{0}-\varepsilon^{2} g_{0}
$$


We use the notation

$$
F(x, \varepsilon)=\lim _{\eta \backslash 0} F(x+i \eta, \varepsilon) \quad \text { and } \quad H_{s}(x, \varepsilon)=\lim _{\eta \searrow 0} H_{s}(x+i \eta, \varepsilon),
$$

whenever these limits exist.

Proposition 2.4. For $E \geq-a / 2$ and $\varepsilon$ sufficiently small the equations

$$
F(x, \varepsilon)=0 \quad \text { and } \quad H_{s}(x, \varepsilon)=0
$$

on $(-a, 0)$ and $(-\infty, 0)$ have unique solutions $x_{b}$ and $\tilde{x}_{b}$, respectively, and we have that

$$
\left|x_{b}-\tilde{x}_{b}\right| \lesssim \varepsilon^{2} \min \left\{\left|\tilde{x}_{b}\right|^{1 / 2},\left|x_{b}\right|^{1 / 2}\right\} .
$$

The main estimation in the singular case is contained in the following Theorem.

Theorem 2.5. Suppose $E \in\left[-a / 2,(c / 2) \varepsilon^{4 / 5}\right]$. Then for all $t \geq 0$ and sufficiently small $\varepsilon$ we have

$$
\left|A_{\varepsilon}(t)-\frac{2 v(f)^{3 / 2}}{2 v(f)^{3 / 2}+1} e^{-i t x_{b}}-\frac{1}{\pi} \int_{0}^{\infty} \frac{y^{1 / 2}}{y(f-y)^{2}+1} e^{-i s y} d y\right| \lesssim \varepsilon^{4 / 5},
$$

where

$$
s=\left(\varepsilon^{2} g_{-1}\right)^{2 / 3} t \quad \text { and } \quad f=\left(\varepsilon^{2} g_{-1}\right)^{-2 / 3} E,
$$

and $v(f)$ is the (unique) solution of

$$
f+v-\frac{1}{v^{1 / 2}}=0
$$

on $(0, \infty)$.

The only quantity in (2.23), which is not expressed in terms of the "model" function $H_{s}(z, \varepsilon)$, and then not explicitly computable, is $x_{b}$. So a natural question is to estimate also the error when replacing $x_{b}$ by $\tilde{x}_{b}$ in (2.23), which amounts to estimate $\left|e^{-i t x_{b}}-e^{-i t \tilde{x}_{b}}\right|$. While $\left|e^{-i t x_{b}}-e^{-i t \tilde{x}_{b}}\right|$ cannot be small uniformly for $t \geq 0$, it is however small on not too long time scales. More precisely, we have the following result. Note that $t \tilde{x}_{b}=-s v(f)$, see (3.19).

Corollary 2.6. Suppose $-\varepsilon^{4 / 3} \lesssim E \lesssim \varepsilon^{4 / 5}$. Then for all $t>0$ and sufficiently small $\varepsilon$ we have

$$
\left|A_{\varepsilon}(t)-\frac{2 v(f)^{3 / 2}}{2 v(f)^{3 / 2}+1} e^{i s v(f)}-\frac{1}{\pi} \int_{0}^{\infty} \frac{y^{1 / 2}}{y(f-y)^{2}+1} e^{-i s y} d y\right| \lesssim \varepsilon^{4 / 5}+s \varepsilon^{4 / 3} .
$$


2.2 The regular case: $g_{-1}=0, g_{1}<0$.

As the model function we take

$$
H_{r}(z, \varepsilon)=E_{0}-z-\varepsilon^{2}\left(g_{0}-i g_{1} \sqrt{z}-g_{2} z\right)=b\left(\tilde{E}-z+i \tilde{g}_{1} \sqrt{z}\right),
$$

where

$$
b=1-\varepsilon^{2} g_{2}, \quad \tilde{E}=\frac{E_{0}-\varepsilon^{2} g_{0}}{b}, \quad \text { and } \quad \tilde{g}_{1}=\frac{g_{1}}{b} .
$$

In what follows $\varepsilon$ is assumed to be sufficiently small, such that $b$ is close to one.

We use the notation from (2.20) for $H_{r}(z, \varepsilon)$, too.

Proposition 2.7. (i) For $\tilde{E} \geq 0$ and $\varepsilon$ sufficiently small we have that $F(x, \varepsilon)>0$ on $(-a, 0)$, and $H_{r}(x, \varepsilon)>0$ on $(-\infty, 0)$.

(ii) For $-a / 2 \leq \tilde{E}<0$ and $\varepsilon$ sufficiently small the equations

$$
F(x, \varepsilon)=0, \quad \text { and } \quad H_{r}(x, \varepsilon)=0
$$

on $(-a, 0)$ and $(-\infty, 0)$ have unique solutions $x_{b}$ and $\tilde{x}_{b}$, respectively, and we have that

$$
\left|x_{b}-\tilde{x}_{b}\right| \lesssim \varepsilon^{2} \min \left\{\left|\tilde{x}_{b}\right|^{3 / 2},\left|x_{b}\right|^{3 / 2}\right\} .
$$

The analogue of Theorem 2.5 can be stated as follows.

Theorem 2.8. Suppose $\tilde{E} \in\left[-a / 2,(c / 2) \varepsilon^{3 / 4}\right]$. Then for all $t \geq 0$, and for sufficiently small $\varepsilon$, we have

(i) For $\tilde{E} \geq 0$ we have

$$
\left|A_{\varepsilon}(t)-\frac{1}{\pi} \int_{0}^{\infty} \frac{y^{1 / 2}}{(\tilde{f}-y)^{2}+y} e^{-i \tilde{s} y} d y\right| \lesssim \varepsilon^{4 / 3}
$$

where

$$
\tilde{s}=\left(\varepsilon^{2} \tilde{g}_{1}\right)^{2} t \quad \text { and } \quad \tilde{f}=\left(\varepsilon^{2} \tilde{g}_{1}\right)^{-2} \tilde{E} .
$$

(ii) For $\tilde{E} \leq 0$ we have

$$
\left|A_{\varepsilon}(t)-\frac{\sqrt{1+4|\tilde{f}|}-1}{\sqrt{1+4|\tilde{f}|}} e^{-i t x_{b}}-\frac{1}{\pi} \int_{0}^{\infty} \frac{y^{1 / 2}}{(\tilde{f}-y)^{2}+y} e^{-i \tilde{s} y} d y\right| \lesssim \varepsilon^{4 / 3} .
$$

As in the singular case one can replace $x_{b}$ by $\tilde{x}_{b}$ in Theorem 2.8 at the price of an error term, which is small on relevant time scales. We let $\tilde{v}(\tilde{f})$ denote the unique positive solution to the equation $\tilde{f}+v+v^{1 / 2}=0$. We have $\tilde{v}(\tilde{f})=\frac{1}{4}(\sqrt{1+4|\tilde{f}|}-1)^{2}$ and $\tilde{x}_{b}=-\left(\varepsilon^{2} \tilde{g}_{1}\right)^{2} \tilde{v}(\tilde{f})$. 
Corollary 2.9. Suppose $-\varepsilon^{4} \lesssim E<0$. Then for all $t>0$ and sufficiently small $\varepsilon$ we have

$$
\left|A_{\varepsilon}(t)-\frac{\sqrt{1+4|\tilde{f}|}-1}{\sqrt{1+4|\tilde{f}|}} e^{i \tilde{s} \tilde{v}(\tilde{f})}-\frac{1}{\pi} \int_{0}^{\infty} \frac{y^{1 / 2}}{(f-y)^{2}+y} e^{-i \tilde{s} y} d y\right| \lesssim \varepsilon^{4 / 3}+\tilde{s} \varepsilon^{4} .
$$

\subsection{The smooth case: $g_{-1}=g_{1}=0$.}

In this case Assumption 2.3 implies that $g(z)$ is uniformly Lipschitz continuous in $D_{a}$, so that one can apply [13, Theorem 4.1] to obtain the following result. See also the recent paper [28], where the analytical continuation approach is used.

Theorem 2.10. Suppose $E_{0} \in[-a / 2, a / 2]$. Write $F(x+i 0, \varepsilon)=R(x, \varepsilon)+$ $i I(x, \varepsilon)$. Then for $\varepsilon$ sufficiently small there exists a unique solution to $R(x, \varepsilon)=$ 0 in the interval $(-a, a)$, denoted by $x_{0}(\varepsilon)$. Let $\Gamma(\varepsilon)=I\left(x_{0}(\varepsilon), \varepsilon\right)$.

Then for $\varepsilon$ sufficiently small, and for all $t>0$, the following result holds true:

$$
\left|A_{\varepsilon}(t)-e^{-i t\left(x_{0}(\varepsilon)-i \Gamma(\varepsilon)\right)}\right| \lesssim \varepsilon^{2}|\ln \varepsilon|
$$

\subsection{Some examples of non-exponential decay laws}

The integrals

$$
I_{s}(f, s)=\frac{1}{\pi} \int_{0}^{\infty} \frac{y^{1 / 2}}{y(f-y)^{2}+1} e^{-i s y} d y
$$

and

$$
I_{r}(\tilde{f}, \tilde{s})=\frac{1}{\pi} \int_{0}^{\infty} \frac{y^{1 / 2}}{(\tilde{f}-y)^{2}+y} e^{-i \tilde{s} y} d y
$$

give the decay laws in the singular and regular cases, respectively. These integrals can be expressed in closed form in terms of special functions, at least in some cases. As an example we mention that one can obtain

$$
I_{r}(0, \tilde{s})=e^{i \tilde{s}}(1-\Phi(\sqrt{i \tilde{s}})) .
$$

Here $\Phi$ denotes the error function. See [7, Formula 3.446.1] and use the Lebesgue dominated convergence theorem.

Thus one may use the properties of the error function to study some asymptotics. Postponing this detailed study to a future publication here we give the results of the direct numerical integration of (2.36) and (2.37) for a 
few values of $f$ and $\tilde{f}$. The details of the computations and the figures are collected in Section 4. The main conclusions are as follows.

(a) For values of $f, \tilde{f}$ of order one (say $|f|,|\tilde{f}| \leq 5$ ), the decay law is definitely non-exponential. In particular for negative values one sees the interference between the bound state contribution and the continuum contribution. See Figure 1, Figure 4, and Figure 5.

(b) As $f, \tilde{f} \rightarrow \infty$ the decay law quickly becomes exponential (at $f=30$ it is hard to distinguish between the numerical result and an exponential), see Figure 2. For $\tilde{f}=30$ the exponential decay takes place very rapidly, the values become very small, and a different behavior is observed, see Figure 6 . Note that in Figure 2 we consider values of $s$ up to 20, whereas in Figure 6 we stop at $\tilde{s}=5$.

(c) As $f, \tilde{f} \rightarrow-\infty$ the survival probability amplitude reaches the bound state form. For $f=-30$, see Figure 3, and for $\tilde{f}=-30$, see Figure 7 . Note the vertical scale on these two figures. They show that the bound state behavior is observed almost instantaneously in the regular case, whereas in the singular case oscillations persist, although with a rapidly decaying amplitude.

It follows that as $E_{0}$ passes through the threshold, the non-exponential decay takes place in a window of energies (see (2.24) and (2.32)) with width of order $\varepsilon^{4 / 3}$ in the singular case and $\varepsilon^{4}$ in the regular case.

Comparing the various results illustrated in the Figures, it is remarkable that such a variety of different decay laws are described by the integrals (2.36) and (2.37).

\section{The proofs}

Proof of Proposition 2.4. The existence and uniqueness of the solutions follow from the fact that for sufficiently small $\varepsilon$ both functions are positive at $x=-a$, tend to $-\infty$ as $x \nearrow 0$ and their derivatives are smaller than -1 . Suppose now that $F\left(x_{b}, \varepsilon\right)=0$. Then from (2.12) it follows that

$$
\left|H_{s}\left(x_{b}, \varepsilon\right)\right|=\left|H_{s}\left(x_{b}, \varepsilon\right)-F\left(x_{b}, \varepsilon\right)\right| \lesssim \varepsilon^{2}\left|x_{b}\right|^{1 / 2} .
$$

Since on $(-a, 0) \frac{d}{d x} H_{s}(x, \varepsilon) \leq-1$, the estimate (3.1) implies

$$
\left|x_{b}-\tilde{x}_{b}\right| \lesssim \varepsilon^{2}\left|x_{b}\right|^{1 / 2}
$$

Repeating the argument with $H_{s}(x, \varepsilon)$ replaced by $F(x, \varepsilon)$, one obtains also that

$$
\left|x_{b}-\tilde{x}_{b}\right| \lesssim \varepsilon^{2}\left|\tilde{x}_{b}\right|^{1 / 2}
$$

and the proof is finished. 
Proof of Theorem 2.5. Since the proof is somewhat complicated, we divide it into a number of steps. Let $I(\varepsilon)=\left[-a, c \varepsilon^{4 / 5}\right], I_{+}(\varepsilon)=\left[0, c \varepsilon^{4 / 5}\right]$, and

$$
\tilde{A}(t)=\lim _{\eta \searrow 0} \frac{1}{\pi} \int_{I(\varepsilon)} d x e^{-i t x} \operatorname{Im} F(x+i \eta, \varepsilon)^{-1} .
$$

We will use the notation introduced in (2.20), and the notation

$$
\frac{d}{d x} F(x, \varepsilon)=F^{\prime}(x, \varepsilon),
$$

provided the derivative exists. From Proposition 2.4, the residue theorem, and the fact that due to Assumption 2.3 one can take the limit $\eta \searrow 0$, one obtains:

$$
\tilde{A}(t)=-\frac{1}{F^{\prime}\left(x_{b}, \varepsilon\right)} e^{-i t x_{b}}+\frac{1}{\pi} \int_{I_{+}(\varepsilon)} d x e^{-i t x} \operatorname{Im} F(x, \varepsilon)^{-1}
$$

Now

$$
\begin{aligned}
\mid \tilde{A}(t)+ & \frac{e^{-i t x_{b}}}{H_{s}^{\prime}\left(\tilde{x}_{b}, \varepsilon\right)}-\frac{1}{\pi} \int_{0}^{\infty} d x e^{-i t x} \operatorname{Im} H_{s}(x, \varepsilon)^{-1} \mid \\
\leq & \frac{1}{\pi} \int_{I_{+}(\varepsilon)} d x \frac{\left|F(x, \varepsilon)-H_{s}(x, \varepsilon)\right|}{|F(x, \varepsilon)|\left|H_{s}(x, \varepsilon)\right|}+\frac{1}{\pi} \int_{c \varepsilon^{4 / 5}}^{\infty} d x \frac{\operatorname{Im} H_{s}(x, \varepsilon)}{\left|H_{s}(x, \varepsilon)\right|^{2}} \\
& +\left|\frac{1}{F^{\prime}\left(x_{b}, \varepsilon\right)}-\frac{1}{H_{s}^{\prime}\left(\tilde{x}_{b}, \varepsilon\right)}\right| .
\end{aligned}
$$

We now estimate the terms (3.4) and(3.5). We begin with the error coming from the replacement of $F(x, \varepsilon)$ by $H_{s}(x, \varepsilon)$ in the integral on $I_{+}(\varepsilon)$.

$$
\begin{aligned}
|F(x, \varepsilon)| & \geq \frac{1}{2}\left|H_{s}(x, \varepsilon)\right|+\frac{1}{2} \operatorname{Im} H_{s}(x, \varepsilon)-\varepsilon^{2} x^{3 / 2}|r(\kappa)|-x^{1 / 2}\left|g_{1}\right| \\
& =\frac{1}{2}\left|H_{s}(x, \varepsilon)\right|+\varepsilon^{2} x^{-1 / 2}\left(\frac{1}{2} g_{-1}-x^{2}|r(\kappa)|-x\left|g_{1}\right|\right),
\end{aligned}
$$

so that for sufficiently small $\varepsilon$ and for $x \in I_{+}(\varepsilon)$ we have

$$
|F(x, \varepsilon)| \geq \frac{1}{2}\left|H_{s}(x, \varepsilon)\right| \text {. }
$$

Accordingly

$$
\begin{aligned}
\int_{I_{+}(\varepsilon)} d x \frac{\left|F(x, \varepsilon)-H_{s}(x, \varepsilon)\right|}{|F(x, \varepsilon)|\left|H_{s}(x, \varepsilon)\right|} & \lesssim \varepsilon^{2} \int_{I_{+}(\varepsilon)} d x \frac{x^{1 / 2}}{(x-E)^{2}+\varepsilon^{4} x^{-1}} \\
& \lesssim \varepsilon^{2+\frac{4}{10}} \int_{0}^{\infty} d x \frac{1}{(x-E)^{2}+\varepsilon^{4-\frac{4}{5}}} \lesssim \varepsilon^{4 / 5}
\end{aligned}
$$


We now estimate the integral

$$
\frac{1}{\pi} \int_{c \varepsilon^{4 / 5}}^{\infty} d x \frac{\left|\operatorname{Im} H_{s}(x, \varepsilon)\right|}{\left|H_{s}(x, \varepsilon)\right|^{2}}
$$

Since by assumption $E \leq(c / 2) \varepsilon^{4 / 5}$ and $x \geq c \varepsilon^{4 / 5}$ one has $(x-E) \geq x / 2$ and then

$$
\begin{aligned}
\int_{c \varepsilon^{4 / 5}}^{\infty} d x \frac{\left|\operatorname{Im} H_{s}(x, \varepsilon)\right|}{\left|H_{s}(x, \varepsilon)\right|^{2}} & \lesssim \varepsilon^{2} \int_{c \varepsilon^{4 / 5}}^{\infty} d x \frac{x^{-1 / 2}}{(x-E)^{2}} \\
& \lesssim \varepsilon^{2} \int_{c \varepsilon^{4 / 5}}^{\infty} d x x^{-5 / 2} \lesssim \varepsilon^{4 / 5}
\end{aligned}
$$

The estimation of

$$
\left|\frac{1}{F^{\prime}\left(x_{b}, \varepsilon\right)}-\frac{1}{H_{s}^{\prime}\left(\tilde{x}_{b}, \varepsilon\right)}\right|
$$

is a bit more complex, so we state it as a lemma.

\section{Lemma 3.1.}

$$
\left|\frac{1}{F^{\prime}\left(x_{b}, \varepsilon\right)}-\frac{1}{H_{s}^{\prime}\left(\tilde{x}_{b}, \varepsilon\right)}\right| \lesssim \varepsilon^{4 / 3}
$$

Proof. We estimate the l.h.s. of (3.10) in two steps, splitting the estimate as shown here.

$$
\begin{aligned}
\left|\frac{1}{F^{\prime}\left(x_{b}, \varepsilon\right)}-\frac{1}{H_{s}^{\prime}\left(\tilde{x}_{b}, \varepsilon\right)}\right| \leq & \left|\frac{1}{F^{\prime}\left(x_{b}, \varepsilon\right)}-\frac{1}{H_{s}^{\prime}\left(x_{b}, \varepsilon\right)}\right| \\
& +\left|\frac{1}{H_{s}^{\prime}\left(x_{b}, \varepsilon\right)}-\frac{1}{H_{s}^{\prime}\left(\tilde{x}_{b}, \varepsilon\right)}\right| .
\end{aligned}
$$

Consider the term on the r.h.s. of (3.11). From Assumption (2.3) it follows that for $x \in[-a, 0)$ we have $\left|F^{\prime}(x, \varepsilon)-H_{s}^{\prime}(x, \varepsilon)\right| \lesssim \varepsilon^{2}|x|^{-1 / 2}$, which together with $\left|F^{\prime}(x, \varepsilon)\right| \geq 1$ gives

$$
\left|\frac{1}{F^{\prime}\left(x_{b}, \varepsilon\right)}-\frac{1}{H_{s}^{\prime}\left(x_{b}, \varepsilon\right)}\right| \lesssim \frac{\varepsilon^{2}\left|x_{b}\right|^{-1 / 2}}{1+\varepsilon^{2}\left|x_{b}\right|^{-3 / 2}}=\varepsilon^{4 / 3} \frac{y}{1+y^{3}},
$$

where $y=\varepsilon^{2 / 3}\left|x_{b}\right|^{-1 / 2}$. Since the function $y /\left(1+y^{3}\right)$ is uniformly bounded on $(0, \infty)$, one obtains

$$
\left|\frac{1}{F^{\prime}\left(x_{b}, \varepsilon\right)}-\frac{1}{H_{s}^{\prime}\left(x_{b}, \varepsilon\right)}\right| \lesssim \varepsilon^{4 / 3}
$$

Consider now the term (3.12). From Taylor's theorem we get (with a slight abuse of notation)

$$
\left|\frac{1}{H_{s}^{\prime}\left(x_{b}, \varepsilon\right)}-\frac{1}{H_{s}^{\prime}\left(\tilde{x}_{b}, \varepsilon\right)}\right| \lesssim \frac{\left|H_{s}^{\prime \prime}(u, \varepsilon)\right|}{\left|H_{s}^{\prime}(u, \varepsilon)\right|^{2}}\left|\tilde{x}_{b}-x_{b}\right|,
$$


where $u$ lies between $x_{b}$ and $\tilde{x}_{b}$. Now from Proposition 2.4

$$
\left|x_{b}-\tilde{x}_{b}\right| \lesssim \varepsilon^{2} \min \left\{\left|\tilde{x}_{b}\right|^{1 / 2},\left|x_{b}\right|^{1 / 2}\right\} \leq \varepsilon^{2}|u|^{1 / 2},
$$

and then

$$
\frac{\left|H_{s}^{\prime \prime}(u, \varepsilon)\right|}{\left|H_{s}^{\prime}(u, \varepsilon)\right|^{2}}\left|\tilde{x}_{b}-x_{b}\right| \lesssim \frac{\varepsilon^{4}|u|^{-2}}{1+\varepsilon^{4}|u|^{-3}}=\varepsilon^{4 / 3} \frac{y^{2}}{1+y^{3}},
$$

where $y=\varepsilon^{4 / 3}|u|^{-1}$. Since the function $y^{2} /\left(1+y^{3}\right)$ is uniformly bounded on $(0, \infty)$, one obtains from (3.15) and (3.16) the estimate

$$
\left|\frac{1}{H_{s}^{\prime}\left(x_{b}, \varepsilon\right)}-\frac{1}{H_{s}^{\prime}\left(\tilde{x}_{b}, \varepsilon\right)}\right| \lesssim \varepsilon^{4 / 3} .
$$

Putting together (3.14) and (3.17), one obtains (3.10) and the proof of the lemma is finished.

From (3.5), (3.10), (3.9), and Lemma 3.1, one obtains (uniformly for $t>0)$ the estimate

$$
\left|\tilde{A}(t)+\frac{e^{-i t x_{b}}}{H_{s}^{\prime}\left(\tilde{x}_{b}, \varepsilon\right)}+\frac{1}{\pi} \int_{0}^{\infty} d x e^{-i t x} \frac{\operatorname{Im} H_{s}(x, \varepsilon)}{\left|H_{s}(x, \varepsilon)\right|^{2}}\right| \lesssim \varepsilon^{4 / 5}
$$

The approximant of $\tilde{A}(t)$ in (3.18) has a nice form in scaled parameters. Using the change of variables $x=\left(\varepsilon^{2} g_{-1}\right)^{2 / 3} y$, the integral takes the form

$$
-\int_{0}^{\infty} d x e^{-i t x} \frac{\operatorname{Im} H_{s}(x, \varepsilon)}{\left|H_{s}(x, \varepsilon)\right|^{2}}=\int_{0}^{\infty} \frac{y^{1 / 2}}{y(f-y)^{2}+1} e^{-i s y} d y
$$

where $s=\left(\varepsilon^{2} g_{-1}\right)^{2 / 3} t$. Furthermore, if

$$
\left|\tilde{x}_{b}\right|=\left(\varepsilon^{2} g_{-1}\right)^{2 / 3} v(f)
$$

then $v(f)$ is the (unique) solution of

$$
f+v-\frac{1}{v^{1 / 2}}=0
$$

on $(0, \infty)$, and

$$
\frac{1}{H_{s}^{\prime}\left(\tilde{x}_{b}, \varepsilon\right)}=-\frac{2 v(f)^{3 / 2}}{2 v(f)^{3 / 2}+1}
$$

Accordingly, (3.18) can be rewritten as

$$
\left|\tilde{A}(t)-\frac{2 v(f)^{3 / 2}}{2 v(f)^{3 / 2}+1} e^{-i t x_{b}}-\frac{1}{\pi} \int_{0}^{\infty} \frac{y^{1 / 2}}{y(f-y)^{2}+1} e^{-i s y} d y\right| \lesssim \varepsilon^{4 / 5} .
$$

By comparing (2.23) with (3.21) one sees that in order to finish the proof of the theorem we are left with the estimation of $\left|\tilde{A}(t)-A_{\varepsilon}(t)\right|$. 


\section{Lemma 3.2.}

$$
\left|\tilde{A}(t)-A_{\varepsilon}(t)\right| \lesssim \varepsilon^{4 / 5} .
$$

Proof. We shall use the "Hunziker trick", see [9], which in our case amounts to observe that since $\operatorname{Im} F(x+i \eta, \varepsilon)^{-1}>0$ for $\eta>0$, one has

$$
\left|\tilde{A}(t)-A_{\varepsilon}(t)\right| \leq\left|\tilde{A}(0)-A_{\varepsilon}(0)\right|=|\tilde{A}(0)-1| .
$$

Now the key observation is that

$$
\frac{2 v(f)^{3 / 2}}{2 v(f)^{3 / 2}+1}+\frac{1}{\pi} \int_{0}^{\infty} \frac{y^{1 / 2}}{y(f-y)^{2}+1} d y=1,
$$

and then from (3.21)

$$
|\tilde{A}(0)-1| \lesssim \varepsilon^{4 / 5},
$$

which together with (3.22) will finish the proof of the lemma.

It remains to verify (3.23) using the residue theorem. Let $h(\zeta)=f-\zeta-$ $i \frac{1}{\sqrt{\zeta}}$. The function $1 / h(\zeta)$ is analytic in $\mathbf{C} \backslash[0, \infty)$ with the exception of a simple pole at $\zeta=-v(f)$ with residue

$$
\frac{1}{h^{\prime}(v(f))}=-\frac{2 v(f)^{3 / 2}}{2 v(f)^{3 / 2}+1}
$$

Now

$$
\frac{1}{\pi} \int_{0}^{\infty} \frac{y^{1 / 2}}{y(f-y)^{2}+1} d y=\lim _{R \rightarrow \infty} \lim _{\eta \searrow 0} \frac{1}{2 \pi i} \int_{\Gamma_{R, \eta}} \frac{1}{h(\zeta)} d \zeta
$$

where the contour is chosen as follows. $\Gamma_{R, \eta}$ consists of two circular arcs and two line segments. The line segments connect $i \eta$ with $R \sqrt{1+\eta^{2}}+i \eta$ and $-i \eta$ with $R \sqrt{1+\eta^{2}}-i \eta$. The small circular arc has radius $\eta$ and connects $i \eta$ with $-i \eta$, traversing the left half plane. The large circle with radius $R$ connects the points $R \sqrt{1+\eta^{2}}+i \eta$ and $R \sqrt{1+\eta^{2}}-i \eta$ and is positively oriented.

In order to apply the residue theorem one has to compute

$$
\lim _{R \rightarrow \infty} \frac{1}{2 \pi i} \int_{|\zeta|=R} \frac{1}{h(\zeta)} d \zeta=-\lim _{R \rightarrow \infty} \frac{1}{2 \pi i} \int_{|\zeta|=R} \frac{1}{\zeta} \frac{1}{1-\frac{f}{\zeta}+\frac{i}{\zeta \sqrt{\zeta}}} d \zeta=-1
$$

Applying the residue theorem to $1 / h(\zeta)$ and using (3.24) and (3.25), one obtains (3.23).

Now from (3.23) and (3.21) written at $t=0$ one has

$$
|\tilde{A}(0)-1|=\left|\tilde{A}(t)-\frac{2 v(f)^{3 / 2}}{2 v(f)^{3 / 2}+1}-\frac{1}{\pi} \int_{0}^{\infty} \frac{y^{1 / 2}}{y(f-y)^{2}+1} d y\right| \lesssim \varepsilon^{4 / 5}
$$

which together with (3.22) finishes the proof of the lemma. 
To finish the proof of the Theorem we note that from (3.21) and Lemma 3.2 follows that

$$
\begin{aligned}
\mid A_{\varepsilon}(t)- & \frac{2 v(f)^{3 / 2}}{2 v(f)^{3 / 2}+1} e^{-i t x_{b}}-\frac{1}{\pi} \int_{0}^{\infty} \frac{y^{1 / 2}}{y(f-y)^{2}+1} e^{-i s y} d y \mid \\
\leq & \left|\tilde{A}(t)-\frac{2 v(f)^{3 / 2}}{2 v(f)^{3 / 2}+1} e^{-i t x_{b}}-\frac{1}{\pi} \int_{0}^{\infty} \frac{y^{1 / 2}}{y(f-y)^{2}+1} e^{-i s y} d y\right| \\
& +\left|\tilde{A}(t)-A_{\varepsilon}(t)\right| \lesssim \varepsilon^{4 / 3}
\end{aligned}
$$

and the proof of Theorem 2.5 is finished.

Proof of Corollary 2.6. From Proposition 2.4 follows that

$$
\left|e^{-i t x_{b}}-e^{-i t \tilde{x}_{b}}\right| \leq t\left|x_{b}-\tilde{x}_{b}\right| \leq \varepsilon^{2}\left|\tilde{x}_{b}\right|^{1 / 2} t .
$$

Now (3.20) implies that $v(f)$ is a decreasing function of $f$, so that for $E \gtrsim$ $-\varepsilon^{4 / 3}$ we have

$$
0<v(f) \lesssim 1
$$

Combining this result with (3.19) we get

$$
\varepsilon^{2}\left|\tilde{x}_{b}\right|^{1 / 2} t \lesssim \varepsilon^{8 / 3} t \cong \varepsilon^{4 / 3} s,
$$

and the proof is finished.

Proof of Proposition 2.7. Part (i) follows, since $F(x, \varepsilon)$ on $(-a, 0)$ and $H_{r}(x, \varepsilon)$ on $(-\infty, 0)$ are continuous and strictly decreasing, and since $F(0, \varepsilon)=H_{r}(0, \varepsilon)=$ $\tilde{E} \geq 0$.

Consider now part (ii). For sufficiently small $\varepsilon$ we have that $F(-a, \varepsilon)>0$ and $H_{r}(-a, \varepsilon)>0$, and then one follows the proof of Proposition 2.4 with the change that now

$$
\left|H_{r}(x, \varepsilon)-F(x, \varepsilon)\right| \lesssim \varepsilon^{2}|x|^{3 / 2} .
$$

Proof of Theorem 2.8. The proof follows the pattern of the proof of Theorem 2.5. Let us start with part (i). Let

$$
I_{+}(\varepsilon)=\left[0, c \varepsilon^{4 / 3}\right]
$$

and

$$
\begin{aligned}
\tilde{A}(t) & =\lim _{\eta \searrow 0} \frac{1}{\pi} \int_{I(\varepsilon)} d x e^{-i t x} \operatorname{Im} F(x+i \eta, \varepsilon)^{-1} \\
& =\frac{1}{\pi} \int_{I_{+}(\varepsilon)} d x e^{-i t x} \operatorname{Im} F(x, \varepsilon)^{-1} .
\end{aligned}
$$


As in the singular case we estimate

$$
\begin{aligned}
\mid \tilde{A}(t) & +\frac{1}{\pi} \int_{0}^{\infty} d x e^{-i t x} \frac{\operatorname{Im} H_{r}(x, \varepsilon)}{\left|H_{r}(x, \varepsilon)\right|^{2}} \mid \\
& \leq \frac{1}{\pi} \int_{I_{+}(\varepsilon)} d x \frac{\left|F(x, \varepsilon)-H_{r}(x, \varepsilon)\right|}{|F(x, \varepsilon)|\left|H_{r}(x, \varepsilon)\right|}+\frac{1}{\pi} \int_{c \varepsilon^{4 / 3}}^{\infty} d x \frac{\left|\operatorname{Im} H_{r}(x, \varepsilon)\right|}{\left|H_{r}(x, \varepsilon)\right|^{2}}
\end{aligned}
$$

using the convention in (2.20) also for $H_{r}$. Again, for sufficiently small $\varepsilon$ one has

$$
|F(x, \varepsilon)| \geq \frac{1}{2}\left|H_{r}(x, \varepsilon)\right| \gtrsim \sqrt{(x-\tilde{E})^{2}+\varepsilon^{4} x},
$$

and then from Assumption 2.3 follows that

$$
\int_{I_{+}(\varepsilon)} d x \frac{\left|F(x, \varepsilon)-H_{r}(x, \varepsilon)\right|}{|F(x, \varepsilon)|\left|H_{r}(x, \varepsilon)\right|} \lesssim \varepsilon^{2} \int_{I_{+}(\varepsilon)} d x \frac{x^{3 / 2}}{(x-\tilde{E})^{2}+\varepsilon^{4} x} .
$$

The r.h.s. of (3.30) is estimated in the next lemma.

\section{Lemma 3.3.}

$$
\varepsilon^{2} \int_{I_{+}(\varepsilon)} d x \frac{x^{3 / 2}}{(x-\tilde{E})^{2}+\varepsilon^{4} x} \lesssim \varepsilon^{4 / 3}
$$

Proof. Consider first the case $\tilde{E}>\frac{1}{2} \varepsilon^{4}$. One has

$$
(x-\tilde{E})^{2}+\varepsilon^{4} x=\left(x-\tilde{E}+\frac{1}{2} \varepsilon^{4}\right)^{2}+\varepsilon^{4} \tilde{E}-\frac{1}{4} \varepsilon^{8} .
$$

Then, since $\varepsilon^{4} \tilde{E}-\frac{1}{4} \varepsilon^{8} \geq \frac{1}{2} \varepsilon^{4} \tilde{E}$, we get

$$
\begin{aligned}
\varepsilon^{2} \int_{I_{+}(\varepsilon)} \frac{x^{3 / 2}}{(x-\tilde{E})^{2}+\varepsilon^{4} x} d x \lesssim \varepsilon^{8 / 3} \int_{I_{+}(\varepsilon)} \frac{x}{\left(x-\tilde{E}+\frac{1}{2} \varepsilon^{4}\right)^{2}+\frac{1}{2} \varepsilon^{4} \tilde{E}} d x \\
=\varepsilon^{8 / 3} \int_{I_{+}(\varepsilon)} \frac{x-\tilde{E}+\frac{1}{2} \varepsilon^{4}}{\left(x-\tilde{E}+\frac{1}{2} \varepsilon^{4}\right)^{2}+\frac{1}{2} \varepsilon^{4} \tilde{E}} d x \\
\quad+\varepsilon^{8 / 3}\left(\tilde{E}-\frac{1}{2} \varepsilon^{4}\right) \int_{I_{+}(\varepsilon)} \frac{1}{\left(x-\tilde{E}+\frac{1}{2} \varepsilon^{4}\right)^{2}+\frac{1}{2} \varepsilon^{4} \tilde{E}} d x \\
\lesssim \varepsilon^{8 / 3}\left|\ln \varepsilon^{4} \frac{1}{2} \tilde{E}\right|+\varepsilon^{8 / 3} \tilde{E}^{1 / 2} \frac{1}{\varepsilon^{2}} \lesssim \varepsilon^{4 / 3}
\end{aligned}
$$

which proves the lemma for $\tilde{E}>\frac{1}{2} \varepsilon^{4}$. In the last step we used that $\tilde{E} \lesssim \varepsilon^{4 / 3}$. 
Consider now the opposite case, i.e. $\tilde{E} \leq \frac{1}{2} \varepsilon^{4}$. Using the fact that for $x>\varepsilon^{4}$ we have $x-\tilde{E} \geq \frac{1}{2} x$ one has

$$
\begin{aligned}
\varepsilon^{2}\left[\int_{0}^{\varepsilon^{4}}+\int_{\varepsilon^{4}}^{c \varepsilon^{4 / 3}}\right] & \frac{x^{3 / 2}}{(x-\tilde{E})^{2}+\varepsilon^{4} x} d x \\
& \lesssim \frac{1}{\varepsilon^{2}} \int_{0}^{\varepsilon^{4}} x^{1 / 2} d x+\varepsilon^{2} \int_{\varepsilon^{4}}^{c \varepsilon^{4 / 3}} \frac{1}{x^{1 / 2}} d x \lesssim \varepsilon^{4}+\varepsilon^{8 / 3}
\end{aligned}
$$

which gives the required estimate for $\tilde{E} \leq \frac{1}{2} \varepsilon^{4}$.

We turn now to the second term in the r.h.s. of (3.28). Since by assumption $\tilde{E} \leq \frac{1}{2} c \varepsilon^{4 / 3}$, for $x>\frac{1}{2} c \varepsilon^{4 / 3}$ one has $x-\tilde{E} \geq \frac{1}{2} x$, and then

$$
\begin{aligned}
\int_{c \varepsilon^{4 / 3}}^{\infty} d x \frac{\left|\operatorname{Im} H_{r}(x, \varepsilon)\right|}{\left|H_{r}(x, \varepsilon)\right|^{2}} & \lesssim \varepsilon^{2} \int_{c \varepsilon^{4 / 3}}^{\infty} \frac{x^{1 / 2}}{(x-\tilde{E})^{2}+\varepsilon^{4} x} d x \\
& \lesssim \varepsilon^{2} \int_{c \varepsilon^{4 / 3}}^{\infty} \frac{1}{x^{1 / 2}\left(x+\varepsilon^{4}\right)} d x \lesssim \varepsilon^{4 / 3}
\end{aligned}
$$

In the last step the change of variable $x=\varepsilon^{4} u^{2}$ has been used. Plugging (3.30), (3.31), and (3.33) into (3.28), one obtains

$$
\left|\tilde{A}(t)+\frac{1}{\pi} \int_{0}^{\infty} d x e^{-i t x} \frac{\operatorname{Im} H_{r}(x, \varepsilon)}{\left|H_{r}(x, \varepsilon)\right|^{2}}\right| \lesssim \varepsilon^{4 / 3}
$$

Using the change of variables $x=\varepsilon^{4}\left|\tilde{g}_{1}\right|^{2} y$, and recalling that $g_{1}<0,(3.34)$ is rewritten as

$$
\left|\tilde{A}(t)-\frac{1}{\pi b} \int_{0}^{\infty} d x e^{-i u y} \frac{y^{1 / 2}}{(\tilde{f}-y)^{2}+y}\right| \lesssim \varepsilon^{4 / 3} .
$$

where $u$ and $\tilde{f}$ are given by (2.32). Since $|b-1| \lesssim \varepsilon^{2}$ from (3.34) and (3.35), one obtains

$$
\left|\tilde{A}(t)-\frac{1}{\pi} \int_{0}^{\infty} d x e^{-i u y} \frac{y^{1 / 2}}{(\tilde{f}-y)^{2}+y}\right| \lesssim \varepsilon^{4 / 3} .
$$

To obtain the estimate for $A_{\varepsilon}$ from (3.36) by using the Hunziker trick we need the analogue of (3.23) for the regular case, viz.

Lemma 3.4. (i) Let $\tilde{f} \geq 0$. Then

$$
\frac{1}{\pi} \int_{0}^{\infty} d x \frac{y^{1 / 2}}{(\tilde{f}-y)^{2}+y}=1
$$


(ii) Let $\tilde{f}<0$. Then $\tilde{v}(\tilde{f})=\frac{1}{4}(\sqrt{1+4|\tilde{f}|}-1)^{2}$ is the unique positive solution of the equation $\tilde{f}+v+v^{1 / 2}$ and

$$
\frac{\sqrt{1+4|\tilde{f}|}-1}{\sqrt{1+4|\tilde{f}|}}+\frac{1}{\pi} \int_{0}^{\infty} \frac{y^{1 / 2}}{(\tilde{f}-y)^{2}+y} d y=1 .
$$

Proof. One applies the argument leading to (3.23) to the function $h(\zeta)=$ $\tilde{f}-\zeta-\zeta^{1 / 2}$. The difference between (i) and (ii) is that in the second case $h(\zeta)$ has a pole at $\tilde{v}(\tilde{f})$ with residue

$$
-\frac{\sqrt{1+4|\tilde{f}|}-1}{\sqrt{1+4|\tilde{f}|}}
$$

From this point onwards the proof of Theorem 2.8 coincide with the proof of Theorem 2.5.

Proof of Corollary 2.9. The proof of Corollary 2.9 is the same (using Proposition 2.7 instead of Proposition 2.4) as the proof of Corollary 2.6. Details are omitted.

\section{Some numerical results}

In this section we display a number of results obtained by evaluating numerically the expressions approximating $A_{\varepsilon}(t)$. The computations have been performed using the numerical integration facilities in the computer algebra program Maple.

\subsection{The singular case}

The expression we evaluate is the following, see (2.26).

$$
\left|\frac{2 v(f)^{3 / 2}}{2 v(f)^{3 / 2}+1} e^{i s v(f)}+\frac{1}{\pi} \int_{0}^{\infty} \frac{y^{1 / 2}}{y(f-y)^{2}+1} e^{-i s y} d y\right|^{2}
$$

Here $v(f)$ solves the equation

$$
f+v-\frac{1}{v^{1 / 2}}=0 .
$$

The plots in the figures show this quantity as a function of the scaled time parameter $s$ for various values of the scaled energy parameter $f$. In the left 

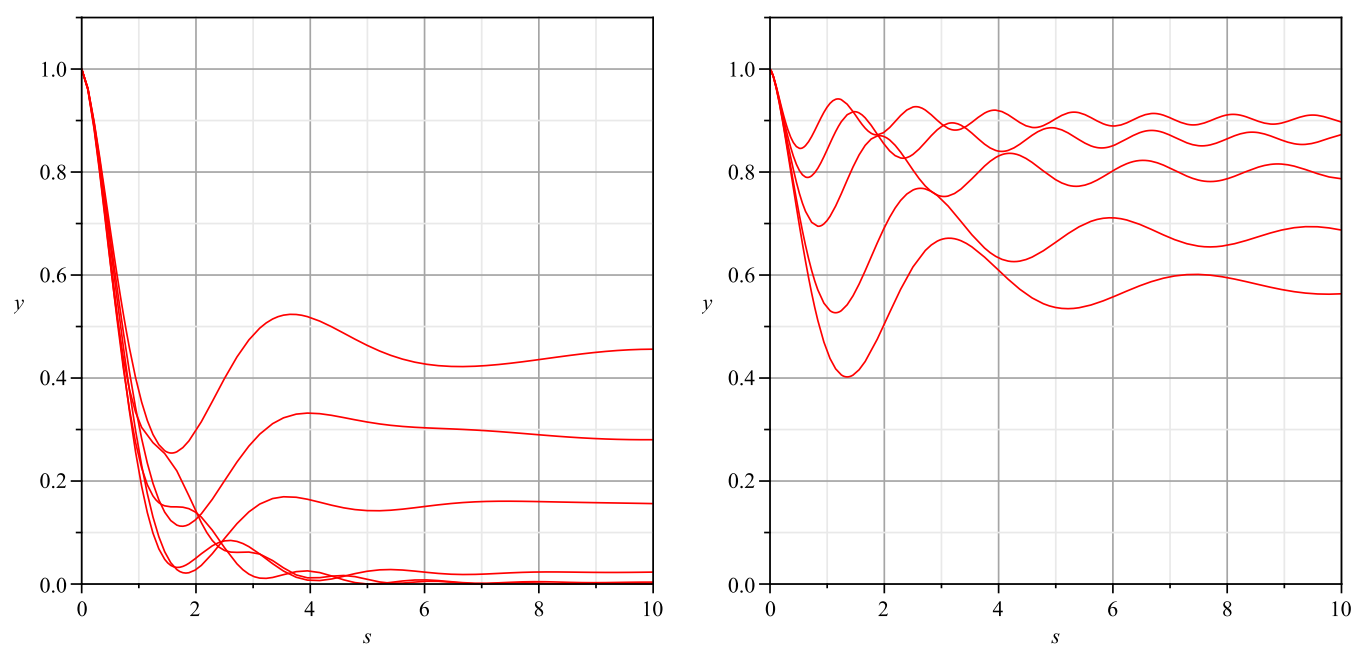

Figure 1: Singular case. On the left the values of the parameter are $f=0$, $0.5,1,2,3,4$, from top to bottom. On the right the values of the parameter are $f=-4,-3,-2,-1,-0.5$, from top to bottom.

hand part of Figure 1 the results are plotted for $f=0,0.5,1,2,3,4$. In the right hand part the results are plotted for the values $f=-0.5,-1,-2,-3$, -4 .

For the value $f=30$ we plot both the value and the logarithm of the value of the quantity in (4.1). The result is shown in Figure 2.

For $f=-30$ the quantity (4.1) is plotted in Figure 3. Note the vertical scale.

\subsection{The regular case}

For positive energy we plot the quantity

$$
\left|\frac{1}{\pi} \int_{0}^{\infty} \frac{y^{1 / 2}}{(\tilde{f}-y)^{2}+y} e^{-i \tilde{s} y} d y\right|^{2} .
$$

The plots in the figures show this quantity as a function of the scaled time parameter $\tilde{s}$ for various values of the scaled energy parameter $\tilde{f}$. In the left hand part of Figure 4 the results are plotted for $\tilde{f}=0,0.5,1,2,3,4$, using a linear vertical scale, and in the right hand part using a logarithmic vertical scale.

For negative values of the energy we plot the quantity

$$
\left|\frac{\sqrt{1+4|\tilde{f}|}-1}{\sqrt{1+4|\tilde{f}|}} e^{i \tilde{s} \tilde{v}(\tilde{f})}+\frac{1}{\pi} \int_{0}^{\infty} \frac{y^{1 / 2}}{(f-y)^{2}+y} e^{-i \tilde{s} y} d y\right|^{2} .
$$



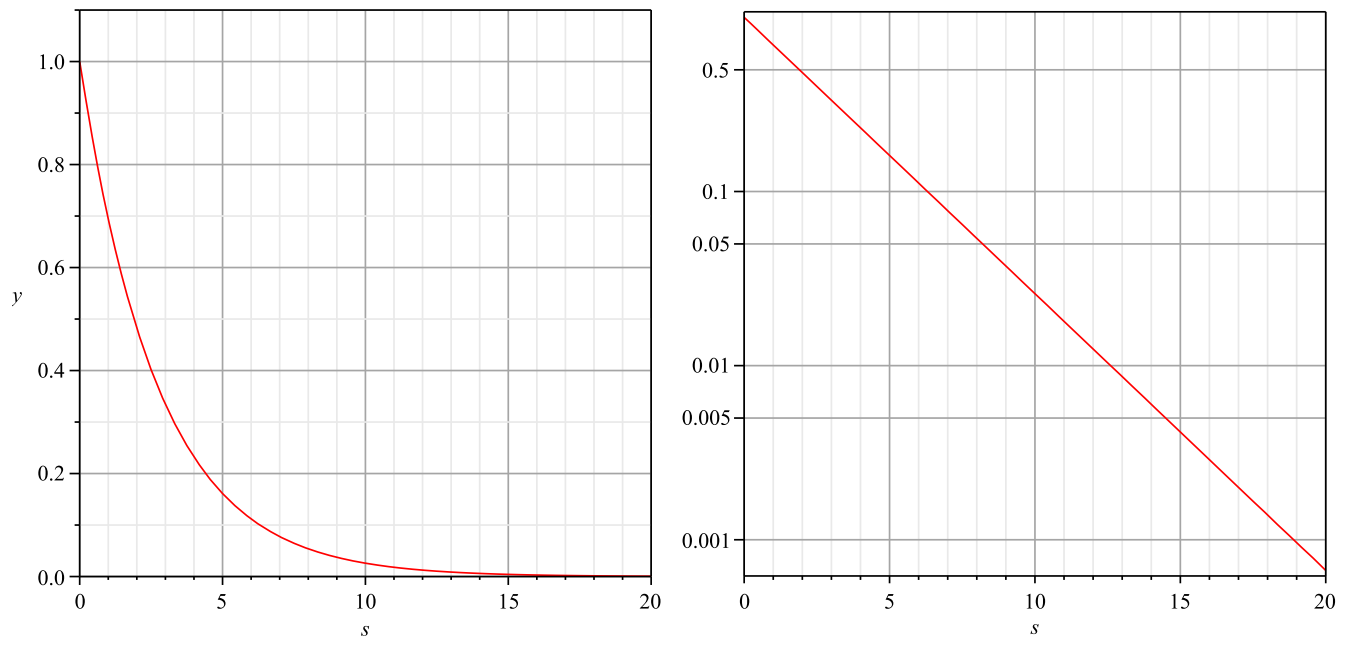

Figure 2: Singular case with the value $f=30$. In the left part the vertical scale is linear, in the right part it is logarithmic.

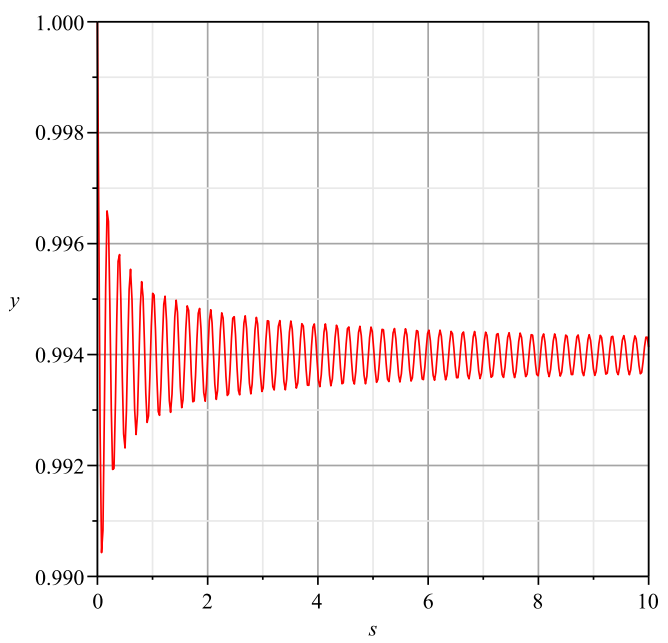

Figure 3: Singular case. Value of the parameter is $f=-30$. Note the vertical scale 

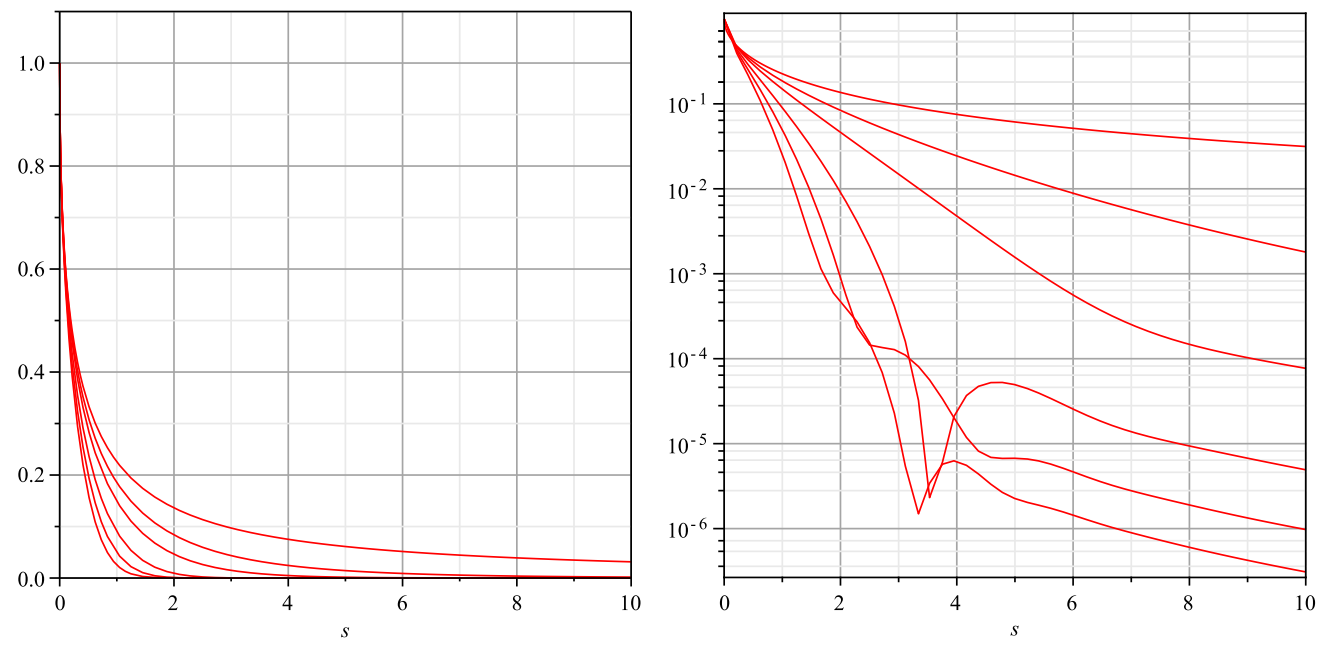

Figure 4: Regular case. The values of the parameter are $\tilde{f}=0,0.5,1,2,3$, 4 , from top to bottom. Left hand part uses a linear vertical scale, right hand part a logarithmic vertical scale.

Here $\tilde{v}(\tilde{f})=\frac{1}{4}(\sqrt{1+4|\tilde{f}|}-1)^{2}$. The plots are shown in Figure 5 for $\tilde{f}=-0.5$, $-1,-2,-3$, and -4 .

For the value $\tilde{f}=30$ we have plotted the quantity (4.2) on both a linear and a logarithmic scale in Figure 6.

For $\tilde{f}=-30$ the quantity (4.3) is plotted in Figure 7 . Note the vertical scale.

\section{Acknowledgments}

A. Jensen was partially supported by the grant Mathematical Physics and Partial Differential Equations from the Danish Natural Science Research Council (FNU).

G. Nenciu was partially supported by Aalborg University. Both this support, and the hospitality of the Department of Mathematical Sciences, Aalborg University, are gratefully acknowledged. 


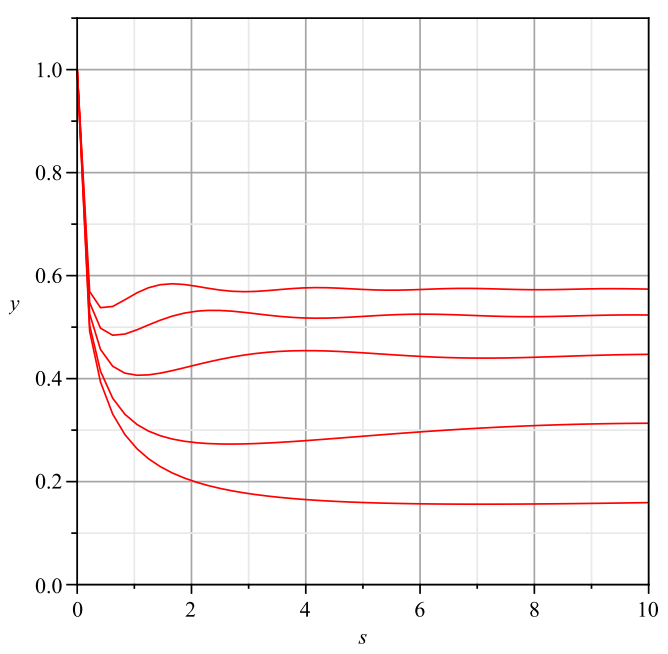

Figure 5: Regular case. The values are $\tilde{f}=-4,-3,-2,-1,-0.5$, from top to bottom.
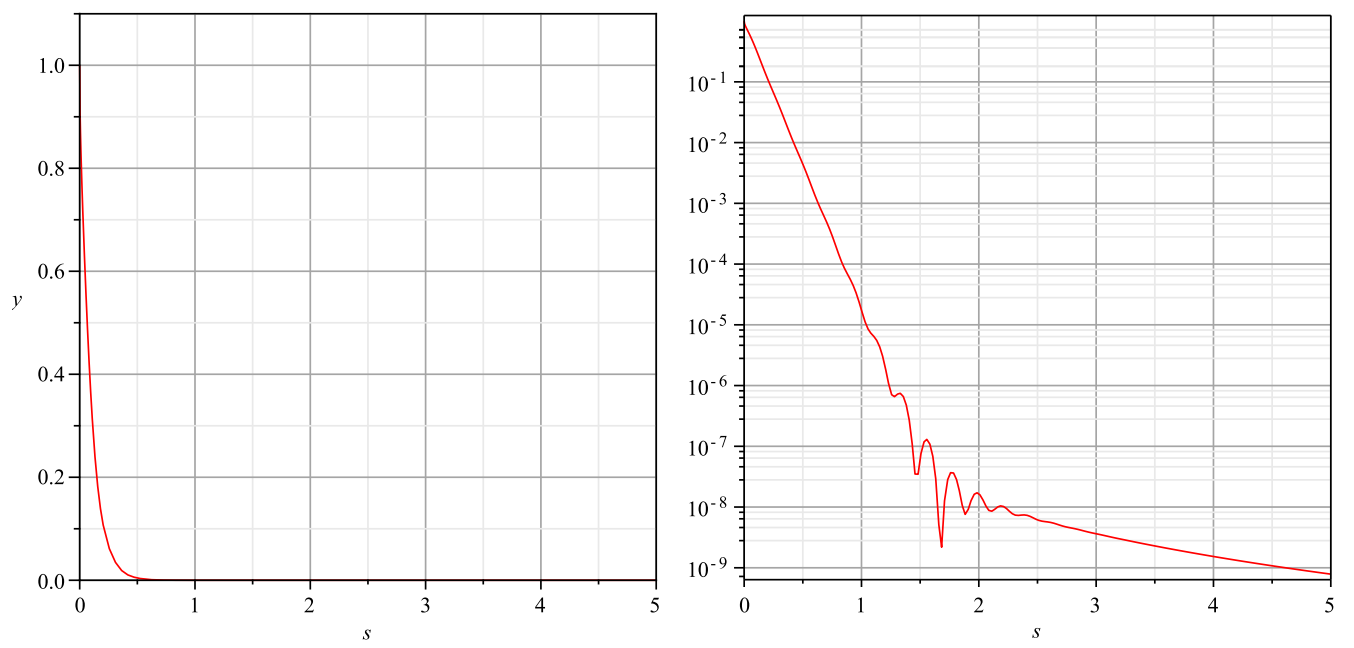

Figure 6: Regular case. The value of the parameter is $\tilde{f}=30$. Left hand plot uses a linear vertical scale, right hand plot uses a logarithmic vertical scale. 


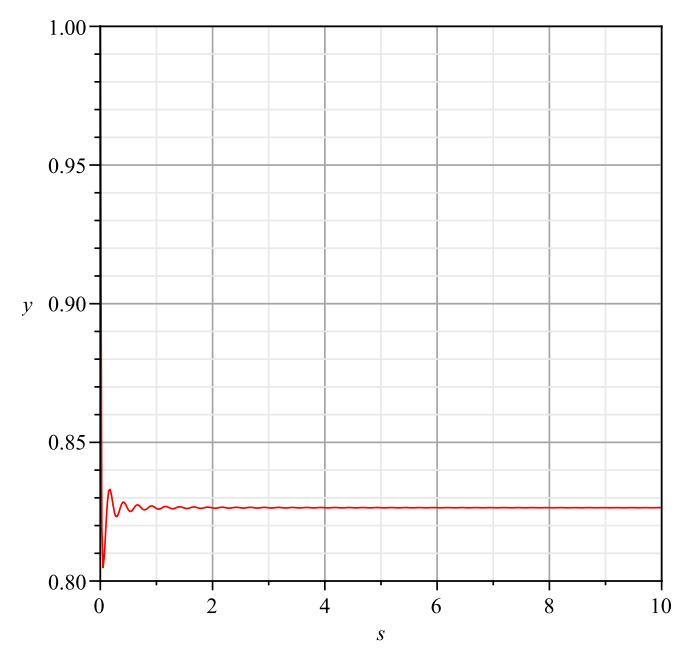

Figure 7: Regular case. Value of the parameter is $\tilde{f}=-30$. Note the vertical scale

\section{A Appendix}

In this Appendix we give some examples of Schrödinger operators satisfying Assumption 2.3. We use results from some of our papers, [13], [14], [10], [11]. In order to verify (2.13) in Assumption 2.3 we use differentiability results for the asymptotic expansions of the resolvent, as given in [11], [10], [22]. We also mention [29], which contains further results on resolvent expansions in the odd dimensional case, and in particular differentiability properties of the expansions. Below we concentrate on the results on the expansion coefficients $g_{j}$ in (2.12).

\section{A.1 Schrödinger operators on the half line}

We refer to [13] and [14] for the details concerning the results presented here. As our first example we take

$$
H_{\mathrm{op}}=-\frac{d^{2}}{d r^{2}}+V(r) \quad \text { on } \quad \mathcal{H}_{\mathrm{op}}=L^{2}([0, \infty)),
$$

with the Dirichlet boundary condition at $r=0$. We will assume that $V$ is real-valued and satisfies

$$
|V(r)| \lesssim(1+r)^{-\beta} \quad \text { for } \quad r \in[0, \infty) .
$$

In the first case to be discussed we assume $\beta>11$. We say that $H_{\mathrm{op}}$ has a zero resonance, if there exists a solution $\Psi_{c} \neq 0$ to $H_{\mathrm{op}} \Psi=0$ (in the sense 
of distributions), such that $\Psi_{c} \in L^{\infty}([0, \infty))$. It is known that for $V$ multiplicative the equation $H_{\mathrm{op}} \Psi=0$ has no solutions belonging to $L^{2}([0, \infty))$. See for example [14, Lemma 3.7]. We assume that $\Psi_{c}$ as been normalized as in [14].

It follows from [14] that Assumption 2.3 is satisfied, if we assume that $W$ is self-adjoint and satisfies

$$
\left|W_{12}(r)\right|+\left|W_{21}(r)\right| \lesssim(1+r)^{-\gamma}
$$

for $\gamma>13 / 4$. Furthermore, we have (using $\langle\cdot, \cdot\rangle$ to denote the pairing between dual spaces as well as the inner product)

$$
g_{-1}=\left|\left\langle W_{12}, \Psi_{c}\right\rangle\right|^{2} \text {. }
$$

Thus if this integral is nonzero, we are in the singular case.

If we assume that $H_{\mathrm{op}} \Psi=0$ has no nonzero solution in $L^{\infty}([0, \infty))$, then we have that $g_{-1}=0$. In that case it suffices to assume $\beta>7$. Furthermore, it suffices to assume $\gamma>9 / 4$. Let us define $G_{0}^{D}$ to be the operator given by the integral kernel

$$
G_{0}^{D}\left(r, r^{\prime}\right)=\min \left\{r, r^{\prime}\right\}
$$

Then we have that

$$
g_{1}=-\left|\left\langle W_{12},\left(I+G_{0}^{D} V\right)^{-1} r\right\rangle\right|^{2}
$$

Thus if this expression is nonzero, then we are in the regular case. If it equals zero, then we are in the smooth case.

For higher angular momenta $\ell \geq 1$ we can take

$$
H_{\mathrm{op}}=-\frac{d^{2}}{d r^{2}}+\frac{\ell(\ell+1)}{r^{2}}+V(r) \quad \text { on } \quad \mathcal{H}_{\mathrm{op}}=L^{2}([0, \infty)),
$$

and in this case no boundary condition is needed at zero. We assume that $V$ decays sufficiently fast at infinity. If zero is not an eigenvalue of $H_{\mathrm{op}}$, then we are in the smooth case. For $V(r)=0$ the details including verification of Assumption 2.3 are in [13]. For general $V$ they have not yet been published. The computations are similar to those in [14].

\section{A.2 Schrödinger operators on $\mathbf{R}^{m}, m$ odd}

As our next example we take

$$
H_{\mathrm{op}}=-\Delta+V(\mathbf{x}) \quad \text { on } \quad \mathcal{H}_{\mathrm{op}}=L^{2}\left(\mathbf{R}^{m}\right),
$$


where we assume that $m$ is odd. Consider first $m \geq 5$. It follows from the results in [10] that Assumption 2.3 is satisfied, if $V$ and $W$ decay sufficiently fast, and if zero is not an eigenvalue of $H_{\mathrm{op}}$. Furthermore, under this assumption and for $m \geq 5$ we are always in the smooth case, i.e. $g_{-1}=0$ and $g_{1}=0$.

Next we consider $m=3$. We say that $H_{\mathrm{op}}$ has a resonance at zero, if there is a solution $\Psi_{c}$ to $(-\Delta+V(\mathbf{x})) \Psi=0$ in the sense of distributions satisfying

$$
\Psi_{c}(\mathbf{x})=\frac{c}{|\mathbf{x}|}+\psi(\mathbf{x}) \quad \text { with } c \neq 0 \text { and } \psi \in L^{2}\left(\mathbf{R}^{3}\right)
$$

Assume that $V$ is real-valued and satisfies

$$
|V(\mathbf{x})| \lesssim(1+|\mathbf{x}|)^{-\beta}
$$

for some $\beta>9$ and furthermore that $W$ is self-adjoint and satisfies

$$
\left|W_{12}(\mathbf{x})\right|+\left|W_{21}(\mathbf{x})\right| \lesssim(1+|\mathbf{x}|)^{-\gamma}
$$

for some $\gamma>5 / 2$.

Assume first that zero is a resonance for $H_{\mathrm{op}}$, but zero in not an eigenvalue. Then the conditions in Assumption 2.3 are satisfied, see [13], [11]. We have that

$$
g_{-1}=\left|\left\langle W_{12}, \Psi_{c}\right\rangle\right|^{2}
$$

see [13, Theorem 5.3]. We assume that $\Psi_{c}$ is normalized, as described in [13]. If $\left\langle W_{12}, \Psi_{c}\right\rangle \neq 0$, then we are in the singular case. If $\left\langle W_{12}, \Psi_{c}\right\rangle=0$, then we may be in the regular case or the smooth case, depending on whether the coefficient $g_{1}$ in this case is non-zero or not. This coefficient is computable, but rather complicated. The expression can be derived from [11].

Next we assume that zero is neither a resonance nor an eigenvalue for $H_{\mathrm{op}}$. Also in this case Assumption 2.3 is satisfied, as a consequence of results in [13], [11]. We have that $g_{-1}=0$ and

$$
g_{1}=-\frac{1}{4 \pi}\left|\left\langle W_{12},\left(I+G_{0}^{0} V\right)^{-1} 1\right\rangle\right|^{2},
$$

where $G_{0}^{0}$ denotes the integral operator with the kernel

$$
G_{0}^{0}(\mathbf{x}, \mathbf{y})=\frac{1}{4 \pi|\mathbf{x}-\mathbf{y}|}
$$

If $\left\langle W_{12},\left(I+G_{0}^{0} V\right)^{-1} 1\right\rangle \neq 0$, then we are in the regular case. Otherwise we are in the smooth case. 
The case $m=1$ can also be treated. In this case zero cannot be an eigenvalue of $H_{\mathrm{op}}$ for a multiplicative potential $V$. If zero is not a resonance, then we are either in the regular case, or in the smooth case. If zero is a resonance, the results are similar to the ones stated above in the case $m=3$. We refer to [13, Theorem 5.6].

\section{References}

[1] L. Cattaneo, G.M. Graf, and W. Hunziker: A general resonance theory based on Mourre's inequality, Ann. H. Poincaré 7 (2006), 583-614.

[2] C.B. Chiu, E.C.G. Sudarshan, and B. Misra: The evolution of unstable quantum states and a resolution of Zeno's paradox. Phys. Rev. D 16, 520-529 (1976).

[3] O. Costin and A. Soffer: Resonance theory for Schrödinger operators, Comm. Math. Phys. 224 , 133-152 (2001).

[4] E.B. Davies: Resonances, spectral concentration and exponential decay. Lett. Math. Phys. 1, 31-35 (1975).

[5] M. Demuth: Pole approximation and spectral concentration, Math. Nachr. 73 (1976), 65-72.

[6] L. Fonda, G.C. Ghirardi, and A. Rimini: Decay theory of unstable quantum systems. Rep. Prog. Phys. 41, 587-631 (1978).

[7] I.S. Gradshteyn and I.M. Ryzhik: Table of Integrals, Series, and Products 5th ed. Academic Press, Boston, 1994.

[8] P.T. Greenland: Seeking the non-exponential decay. Nature 335, 298299 (1988).

[9] W. Hunziker: Resonances, metastable states and exponential decay laws in perturbation theory, Comm. Math. Phys. 132 (1990), 177-188.

[10] A. Jensen: Spectral properties of Schrödinger operators and time-decay of the wave functions. Results in $L^{2}\left(\mathbf{R}^{m}\right), m \geq 5$, Duke Math. J. 47 (1980), 57-80.

[11] A. Jensen and T. Kato: Spectral properties of Schrödinger operators and time-decay of the wave functions, Duke Math. J. 46 (1979), 583-611. 
[12] A. Jensen and G. Nenciu: A unified approach to resolvent expansions at thresholds, Rev. Math. Phys. 13 (2001), no. 6, 717-754.

[13] A. Jensen and G. Nenciu: The Fermi golden rule and its form at thresholds in odd dimensions. Comm. Math. Phys. 261 (2006), 693-727.

[14] A. Jensen and G. Nenciu: Schrödinger operators on the half line: Resolvent expansions and the Fermi golden rule at thresholds, Proc. Indian Acad. Sci. (Math. Sci.) 116 (2006), 375-392.

[15] A. Jensen and G. Nenciu: On the Fermi golden rule: Degenerate eigenvalues, Proc. Conf. Operator Theory and Mathematical Physics, Bucharest, August 2005. To appear.

[16] T. Jittoh, S. Masumoto, J. Sato, Y. Sato, and K. Takeda: Nonexponential decay of an unstable quantum system: Small Q-value s-wave decay. Phys. Rev. A 71, 012109 (2005).

[17] T. Köhler, K. Gòral, and P. Julienne: Production of cold molecules via magnetically tunable Feshbach resonances, Rev. Mod. Phys. 78, 13111361 (2006).

[18] M. Lewenstein, J. Zakrewski, T. Mossberg, and J. Mostowski: Nonexponential spontaneous decay in cavities and waveguides, J. Phys. B: At. Mol. Opt. Phys. 21, L9-L14 (1988).

[19] B. Marcelis, E. van Kempen, B. Verhaar, and J. Kokkelmans: Feshbach resonances with large background scattering length: interplay with open-channel resonances. Phys. Rev. A 70, 012701 (2004).

[20] M. Merkli and I.M. Sigal: A time-dependent theory of quantum resonances, Comm. Math. Phys. 201 (1999), 549-576.

[21] M. Miyamoto: Bound-state eigenenergy outside and inside the continuum for unstable multilevel systems. Phys. Rev. A 72, 063405 (2005)

[22] M. Murata: Asymptotic expansions in time for solutions of Schrödingertype equations, J. Funct. Anal. 49 (1982), no. 1, 10-56.

[23] H. Nakazato, M. Namiki, and S. Pascazio: Temporal behavior of quantum mechanical systems. International J. Modern Physics B 3, 247-295 (1996).

[24] R. G. Newton: Quantum Physics, Springer-Verlag 2002. 
[25] C. Nicolaides: Physical constraints on nonstationary states and nonexponential decay. Phys. Rev. A 66, 022118 (2002)

[26] N. Nygaard, B. Schneider, and P. Julienne: Two-channel R-matrix analysis of magnetic-field-induced Feshbach resonances, Phys. Rev. A 73, 042705 (2006)

[27] A. Orth: Quantum mechanical resonance and limiting absorption: the many body problem, Comm. Math. Phys. 126 (1990), 559-573.

[28] X. P. Wang: Embedded eigenvalues and resonances of Schrödinger operators with two channels, Ann. Fac. Sci. Toulouse Math. (6) 16 (2007), no. $1,179-214$.

[29] K. Yajima: The $L^{p}$ boundedness of wave operators for Schrödinger operators with threshold singularities I, Odd dimensional case, J. Math. Sci. Univ. Tokyo 13 (2006), 43-93. 